\title{
Langues de la cité, langues des cités
}

Jean-Michel Kasbarian

\section{(2) OpenEdition}

Journals

Édition électronique

URL : http://journals.openedition.org/trema/1734

DOI : $10.4000 /$ trema. 1734

ISSN : 2107-0997

\section{Éditeur}

Faculté d'Éducation de l'université de Montpellier

\section{Édition imprimée}

Date de publication : 1 octobre 1999

Pagination : 87-102

ISSN : 1167-315X

\section{Référence électronique}

Jean-Michel Kasbarian, «Langues de la cité, langues des cités », Tréma [En ligne], 15-16| 1999, mis en ligne le 01 octobre 1999, consulté le 30 avril 2019. URL : http://journals.openedition.org/trema/1734 ; DOI : 10.4000/trema. 1734

Ce document a été généré automatiquement le 30 avril 2019

Trema 


\title{
Langues de la cité, langues des cités
}

\author{
Jean-Michel Kasbarian
}

\section{De quoi parle t-on?}

1 Les années quatre-vingt-dix voient se développer dans les médias les discours réifiants sur les «banlieues », le plus souvent stigmatisants, autour du thème de la «violence des jeunes des cités", beaucoup plus rarement valorisants pour ce qui est des réussites individuelles («la sortie de zone» dans le Rap, par exemple), les faits-divers quotidiens constituant pour les journalistes le schéma narratif d'un genre particulier de récit mythique, sorte $\mathrm{d}^{\prime}$ « urban légend » apocalyptique de la zone.

2 La sociologie a fait du "phénomène de société » des banlieues un objet d'étude privilégié mais les études linguistiques se résument jusqu'à présent à quelques articles (Boyer, 1994 ; Kasbarian, 1997, pour un tour d'horizon). L'essentiel de la production autour de cet "objet linguistique médiatiquement identifié» (Boyer) reste encore constitué d'ouvrages grand public (lexiques, recueils de mots et d'expressions) d'abord pour d'évidentes raisons de facilité (la collecte lexicale est plus facile et plus attrayante pour le public que l'analyse d'un corpus sous des angles syntaxiques, morphologiques ou énonciatifs). Mais aussi parce que la mise en scène des mots des variétés «exotiques » de "français des cités » opère sur fond d'emblématisation du «métissage » culturel et langagier, le français contemporain des cités étant alors considéré comme une forme de «créole ». Le travail le plus conséquent sur les pratiques langagières des cités reste à ce jour l'ouvrage d'ethnologie de Lepoutre (1997) ${ }^{1}$.

L'étude des pratiques langagières des cités, de leur rapport au français de référence ( «la langue de la cité ", langue du "vivre ensemble») doit selon nous inclure les trois dimensions de la la délimitation conceptuelle de l'objet (de quoi parle-t-on ?), du champ de l'analyse (comment et quoi décrire?) et de la visée praxéologique qui intéresse particulièrement les participants de cet atelier (comment tenir compte des résultats des recherches pour s'employer à résoudre certains problèmes sociaux, scolaires, culturels ?).

Pour ce qui est de la délimitation conceptuelle de l'objet, on constatera - et l'intitulé de notre atelier "Langues de la cité, langues des cités " y fait implicitement référence - 
qu'elle n'échappe pas à la désormais classique dichotomie Saussurienne de la "langue » et de la "parole $»^{2}$ : sommes-nous en présence d'un français des cités (une langue) ou de pratiques langagières déterminant différents répertoires personnels et collectifs des « jeunes des cités » (plan de la parole)?

Au plan sociolinguistique, la question de la dénomination des «langues ", «langages », "variantes», «dialectes », " parlers », " parlures » des cités est inséparable d'une axiologie stigmatisante ou légitimante des pratiques langagières des cités. La confusion journalistique de la dénomination ${ }^{3}$ des réalités langagières urbaines participe clairement du conflit (repérable aussi bien dans la question des langues régionales, de l'oral...) entre légitimité langagière ("ça existe", "ça correspond à un besoin d'échanges ») et normativité linguistique («ce n'est pas du français», «c'est pauvre...»). La survalorisation de la réalité langagière des cités, effet de catégorisations sociolinguistiques en terme de "richesse ", "fraîcheur ", «ingéniosité ", « créativité " des "mots des jeunes" réifieen même temps qu'elle folkorise (apprivoise) certains aspects (essentiellement lexicaux) de la communication dans les cités, négligeant la dynamique sociale du changement linguistique et l'inscription nécessaire des variantes urbaines dans la question générale de la variabilité du français. Par ailleurs, cette survalorisation communautaire et territorialisante élude la question des limites du " parler des banlieues ", le problème du passage des «langues des cités » à la " langue de la cité ", le français de référence. Parmi les analyses stigmatisantes des parlers des banlieues, on retiendra celle, emblématique, du linguiste Alain Bentolila, spécialiste des questions de lecture et d'illettrisme, évoquant "le faux semblant du français branché» (Le Monde, 1998). Dans des termes que n'aurait pas renié Bernstein, Bentolila oppose le code restreint, territorialisé, identitaire et contextualisé du vernaculaire des jeunes des cités (catégorisé comme " langue terriblement pauvre », " vocabulaire restreint », " langue affaiblie ») à l'universel linguistique du code élaboré, langue de l'école, déter-ritorialisée, universalisante et garante du lien social :

"Confinée dans un espace réduit, rarement sollicitée pour transmettre ou recevoir des informations extérieures à son cercle étroit, c'est une langue terriblement pauvre dont disposent ces citoyens: un vocabulaire restreint, un usage des marques grammaticales très approximatif; une quasi-impossibilité à organiser temporellement des événements. Cette langue affaiblie, quasi exsangue, ne supporte que la proximité, fuit avec effroi et ressentiment toute invitation à fonctionner au large ; elle ferme définitivement les portes $d u$ monde de l'écrit ".

6 Précisément, il nous semble que c'est à la description par l'enquête de terrain de ces «quasi » que devraient s'attacher les linguistes de la réalité urbaine, en s'affranchissant d'un débat dont Labov en son temps étudiant le «VNA ", Vernaculaire Noir Américain (1978), s'était fait le critique, invalidant par la description les concepts de «déficit », « handicap socio-linguistique », " privation verbale » (il est vrai, pour lui opposer dans une vision politique « de gauche », une représentation valorisante du «VNA is beautifull »).

7 Nous proposons dans cet atelier d'introduire le débat sur la question du «nom des langues » par un repérage des attributs sociolinguis-tiques actualisés dans les titres d'une vingtaine d'ouvrages consacrés aux variétés langagières urbaines ( $C f$., tableau ci-dessous et Annexe 1, p. 99), selon les trois catégories, de la dénomination linguistique (DL), du caractère générationnel (CG), et de la territorialisation $(T)$. 


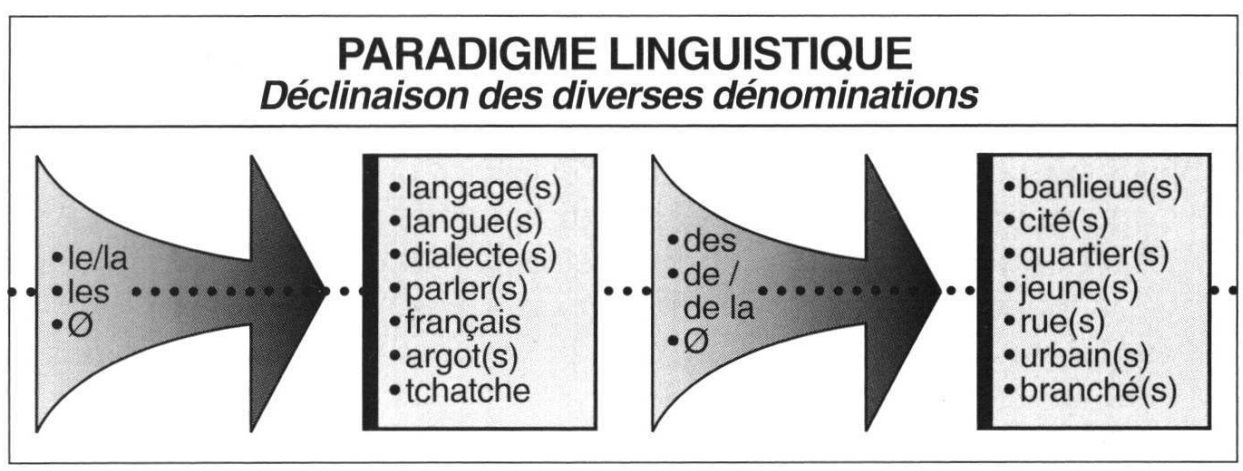

\begin{tabular}{|c|c|c|}
\hline \multicolumn{3}{|c|}{ Catégories d'attributs sociolinguistiques } \\
\hline $\begin{array}{l}\text { Attributs de dénomination linguistique - DL } \\
\text { (Ø) non marqué } \\
\text { (1) langue } \\
\text { (2) langage } \\
\text { (3) dialecte/sabir/créole/argot/tchatche } \\
\text { (4) français (branché/nouveau/contem } \\
\text { porain/des cités/mixte }\end{array}$ & $\begin{array}{l}\text { Attributs du caractère générationnel - CG } \\
\text { (Ø) non marqué } \\
\text { (5) jeune }\end{array}$ & $\begin{array}{l}\text { Attributs de la territorialisation - } T \\
\text { (Ø) non marqué } \\
\text { (6) cité/téci(s) } \\
\text { (7) banlieue/banlieues }\end{array}$ \\
\hline
\end{tabular}

8 On relèvera d'abord que les différentes dénominations se déclinent de façon aléatoire dans un paradigme dont la forme est illustrée par le diagramme ci-dessous.

Une première lecture de cet ensemble met en évidence un facteur chronologique dans les dénominations, celles produites au début de l'observation du phénomène dans les années 1980 et celles où l'objet « langues des cités » occupe le devant de la scène médiatique, les années 1990. Dans la première période, la dénomination du français des cités est essentiellement celle d'un sociolecte générationnel (langage des jeunes, français des jeunes, le jeune, langue ado) ou transgénérationnel (nouveau français, français branché, parlers argotiques contemporains, parlers branchés). Le sociolecte générationnel pose clairement le problème de délimitation des locuteurs : s'agit-il d'une catégorie abstraite, d'un idéal-type ou de groupes spécifiques qu'il faudrait définir en fonction d'un milieu et surtout d'un âge (le passage au Lycée marque un changement significatif des pratiques langagières des adolescents, issus de milieux privilégiés ou de la classe moyenne et usant au collège volontiers du parler banlieue, alors que les élèves des banlieues défavorisées ont plus de difficulté à entrer dans le français de référence). Par ailleurs, l'essentiel n'est pas l'émergence de variantes générationnelles, qui ont toujours existé dans le passé, mais la dynamique propre du changement linguistique des parlures «jeunes» induite par l'appropriation des formes par les médias ${ }^{4}$, formes alors délaissées ou réencodées (verlan de verlan...) comme réponse à la récupération.

Dans la deuxième période des années 1990, la dénomination territoriale s'impose avec la montée de la «violence urbaine » en fait et en représentations ${ }^{5}$, le français des cités apparaissant comme « toposocio-lecte » (langage des banlieues, langage cité, langage téci, langage des Keums, langage quartier).

11 Une deuxième niveau d'interrogation est celui de l'emploi du singulier ou du pluriel dans la dénomination : existe-t-il une pratique qu'une certaine partie de la jeunesse aurait en partage ? y a t-il au contraire plusieurs pratiques, plusieurs langues ou langages ? Comme le remarque Branca-Rosoff $(1996$, p. 83) d'un point de vue théorique, "est-il si aisé de savoir [...] à quel moment un registre informel (le parler des jeunes des banlieues) se constitue en code distinct? ".N'a t-on pas affaire à des pratiques, des productions langagières dans des contextes communicationnels bien spécifiques (au sein du groupe de pairs, dans des 
« interstices » spatio-temporels : cages d'escaliers, bus, couloirs, Cf., Lopez, 1998) avec ce qui peut poser problème - le maintien de ces pratiques dans des situations de plus large communication (école, lieu de travail, bureaux)? C'est ainsi que refusant la tentation « exotique » de la « langue des jeunes », Alain Rey (in, Garcia et Malaurie, 1998, p. 30) en propose la description en terme d'usage langagier : "Le parler des jeunes, ça n'est évidemment pas une langue mais un des usages de la langue »Lepoutre (1997, p. 135) pour sa part, introduit la notion de " performance » qui réfère tout autant à la linguistique qu'aux arts plastiques (la performance comme réalisation artistique provisoire et contextuelle), les usages langagiers s'inscrivant dans une "culture des rues »: "Dans le contexte de la culture des rues, le langage est en effet d'abord conçu et pratiqué comme une performance ».

Autre interrogation: l'inscription des dénominations dans des référents linguistiques constitués permet l'éclairage du "nouveau » par «l'ancien", mais ne va pas sans créer des difficultés ;

- «L'argot » réfère à une fonctionnalité sociale et communicationnelle. Mais la fonction cryptique est dominante dans l'argot, ce qui n'est pas le cas du français contemporain des cités, d'abord identitaire et ludique (Goudaillier, 1997).

- La «tchatche» (un des participants à l'atelier qui a travaillé dans la banlieue de La Paillade à Montpellier, Juan Antonio Lopez parle de «baratin »,terme qu'on retrouve dans la chanson marseillaise récente pour désigner une forme langagière ritualisée, le "mégabaratin »,ou encore de "grillades »ou " d'enfades»).Dans cette domination, le français des cités n'apparaît pas comme un code ou une variante du code mais comme un discours, une forme d'interaction contextuellement pertinente ${ }^{6}$.

- A la dénomination « langue » comme système autonome s'opposent les dénominations de « langage », « parler », " parlure », variantes du code ou moyen linguistique de communication d'une communauté socioculturelle spécifique.

- La dénomination de « dialecte » pour une réalité urbaine confond (tout à la fois éclaire et obscurcit) le dialecte géographique (variété langagière propre à une région, objet de la dialectologie européenne) et le dialecte social (caractérisation d'un groupe social par ses réalisations linguistiques) de la linguistique labovienne.

Pour notre part (Kasbarian, 1997, p. 24), les dénominations qui nous paraissent les plus pertinentes sont celles de "pratiques langagières" (inscrites dans un répertoire linguistique individuel et collectif avec d'autres variétés ou langues, français de scolarisation, langues de l'immigration...) et «de français contemporain des cités » : il s'agit de français et non d'une langue " exotique ", décrit en synchronie, en intégrant la composante topolectale (des cités).

\section{Sur le terrain de la cité / des cités}

$14 \mathrm{Au}$ cours de l'atelier, Nathalie Binisti a présenté quelques éléments pertinents pour le thème à partir de deux enquêtes réalisées auprès de jeunes dits "des quartiers ", à Marseille. La première porte sur les mots et expressions des jeunes dans les quartiers Nord de Marseille et plus précisément autour du parler des jeunes de l'Estaque-gare (les "pasteuriens", du nom de la rue frontière d'un semi-bidonville) et l'autre, sur les pratiques langagières de jeunes filles vivant dans une cité située aussi dans les quartiers nord. 
Les caractéristiques communes des jeunes habitant les «quartiers» populaires de Marseille (le «quartier » ne se confond pas avec la « cités » ou la «banlieue », du fait de frontières territoriales beaucoup moins étanches à Marseille, la circulation entre les « quartiers » et la ville, « en ville » désignant le centre, étant facile). Ce que l'on retrouve néanmoins dans toutes ces situations, c'est le fait que ces jeunes viennent de familles nombreuses, très modestes et de différentes origines. De plus, on peut noter aussi la place particulièrement importante que le groupe de pairs tient dans la vie de ces jeunes du fait de la proximité des habitats, des différentes communautés d'origine et de la vie de quartier. Tous ces éléments se retrouvent dans leur langage sous diverses formes ou expressions qu'une figure emblématique de la vie culturelle marseillaise caractérise de «métissées » ou, de façon polémique, de « créolisées » (Izzo, 1998) :

- un lexique dans lequel se mêlent des mots de plusieurs origines (rom, arabe, maghrébin, kabyle, comorien, anglais, « provençal maritime » (méditerranéen) et parler populaire marseillais doublement minorisés par rapport au français standard et au « provençal pierreux » (latin) de l'intérieur);

- des expressions liées au vécu personnel et à l'expérience de la rue qui relèvent pour l'essentiel du « français contemporain des cités » circulant ;

- des expressions liées à des formes d'interaction adolescente au cours desquelles on a l'impression que le jeune est sans cesse en train de tester ses propres limites mais aussi celles de ceux qui l'entoure, à travers des pratiques verbales identitaires.

L'importance de la fonction ludique avec la fonction identitaire apparaît dans les jeux de langage qui constituent une part importante des pratiques langagières des cités et quartiers de Marseille. Il est possible d'aborder ces jeux de langage du double point de vue formel (structurel) et fonctionnel (sociolinguistique), le second touchant plus particulièrement à la question de la citoyenneté qui nous occupe dans ce colloque. Sur le plan formel, les jeux de langage en usage dans les cités ont contribué de façon significative à l'évolution sémantique du parler marseillais dans les quinze dernières années. Ils se caractérisent par des procédures classiques de glissement de sens et de refonte des signifiants appliquées aux différents composants du lexique disponible, base provençale, argot et parler populaire marseillais, langues de l'immigration, français contemporain des cités. Nous illustrerons ces procédés dans quelques usages qui empruntent à différentes sources.

17 Les glissements de sens sont essentiellement l'effet d'une dérivation métaphorique ou métonymique. Les déplacements de significations peuvent se traduire par un rétrécissement ou un élargissement, selon que le mot passe d'un usage spécialisé à un usage courant ou l'inverse. Exemples de métaphorisation avec rétrécissement de sens de termes provençaux dans un usage qui emprunte au français circulant des cités : en emploi dominant, "amandons» ne désigne plus les "petites amandes » mais les "testicules » ( " tu me tagues les amandons», sur la structure argotique et avec le sens de "casser les couilles ») ; la « chichourle » n'est plus un fruit, la « jujube », mais une « bosse » ou « le sexe de la femme " (" enchaîner la chichourle d'une meuf ", c'est pour un homme « rendre une femme dépendante sexuellement»); dans l'expression dépréciative "être un zeub (" sexe », terme argotique emprunté à l'arabe) de garri », pour " être un moins que rien », le second terme ne désigne plus qu'un «rat» alors qu'il est largement polysémique en provençal.

18 Exemples de métaphorisation avec élargissement du terme provençal et emprunt au français des cités : dans l'expression « une airbag pégueuse " pour « une fille collante », le 
premier terme est en usage dans les banlieues parisiennes alors que le provençal "pégueux» ne désigne plus un "gros berlingot poisseux» mais quelqu'un de "collant » (analogie d'effet). Dans «l'anchoïade en bascour» (" passage à tabac»), le premier terme n'est plus en usage dominant comme désignation culinaire de «l'anchois pilé avec l'aill » mais avec le sens de "bousculade», le second, en emploi circulant désignant un « commissariat».

taphore est dominante pour ce qui est de l'évolution du vocabulaire des cités marseillaises, les significations peuvent devenir périphériques, secondaires ou récessives au terme d'un processus d'équivalence métonymique : l'expression "être à l'agachon » procède d'une métonymie du lieu pour la chose signifiée avec élargissement de sens (l'agachon est un poste de chasse ; "agachonner le schmitt", c'est "être à l'affut" du " policier » ("schmitt » est emprunté au français circulant des cités) quand on réalise un mauvais coup).

Dans les déplacements non métaphoriques avec retrécissement ou élargissement de sens, l'actualisation de traits différentiels à partir d'une signification première peut conduire à son effacement.

21 Rétrécissement au vocabulaire du sexe : «chasper » n'est plus « tâter » mais « palper » (au sens érotique, comme dans "chasper la carrosserie", le second terme désignant la poitrine en argot) ; « monter » dans l'expression « monter en zon'» ce n'est plus pour un Marseillais «quitter Marseille (vers le Nord)» mais «aller en prison» («zonzon» en français cité, située à Luynes, près d'Aix-en-Provence, au Nord de Marseille).

L'évolution sémantique des formes lexicales en usage dans les cités marseillaises se caractérise aussi par des procédés qui remodèlent les signifiants.

Citons ici le procédé de composition. Ainsi, à partir de "collègue", dénomination provençale de «l'ami», les jeunes marseillais ont produit le mot-valise lexicalisé "collèganous", dénomination par auto-référence des «membres de la tribu des marseillais». Avec le français des cités "pourrav» («pourri », avec le suffixe romani «[...]ave»), les jeunes des cités marseillaises composent le désignatif «pourrav de la crêche " ( le ravi de la crêche » désigne le "fada», l'illuminé). Si "meskin » désigne en français des cités un "pauvre type » (emprunt à l'arabe), on trouve en marseillais des cités l'insulte "fatche de mesquin " ( fatche», classique en marseillais, vient de l'italien "faccia », dans des expressions « fatche de... »)

Les néologismes dérivés les plus fréquents sont ceux qui ont trait à la production d'images identitaires. Ainsi, autour du nom «aïoli » et par métaphore sur le plan du signifié (le « piquant » de l'aïoli), on trouve le dénominal « aïoliser » pour « envoûter » et le dérivé nominal "aïolisation " pour " enchantement », la magie étant dans l'usage lié à une expérience bien localisée (l'aïolisation d'un soir de match de l'OM...). "Moulonner ", dénominal de "moulon» («tas» en provençal) est dominant dans l'usage, pour «(s)'entasser » en particulier dans les hauts-lieux de la socialité des jeunes marseillais (on moulonne au stade dans le Virage-Sud, sur la plage du Prado l'été...).

Comme exemple de resuffixation, essentiellement péjorative, on citera "crassous», en emploi nominal et adjectival dans le désignatif "crassous'man» qui dénomme une "personne sale, répugnante", dérivé de "crassir » attesté en provençal avec le sens de « salir» (récessif).

Sur le plan fonctionnel, sociolinguistique, les jeux de langage et plus largement les usages langagiers des cités, posent la question d'une représentation et d'une pratique de la 
citoyenneté dans les images identitaires produites en discours. Ces images nous paraissent s'organiser autour deux axes horizontal et vertical bipolaires. Le premier (axe de catégorisation) procède de l'opposition des pôles de l'autoréférence («nous») et de l'hétéroréference («eux»), le second (axe d'identification) oppose similitude («pareil que ») et différence ("différent de »). Quatre images identitaires sont alors possibles, repérables dans les différentes séquentialités du déroulement de l'échange et que nous proposons d'illustrer d'exemples.

\section{L'identité fusionnelle}

L'identité fusionnelle des membres dominants des cités est le produit d'une catégorisation autoréférentielle et d'une identification par similitude (Moi = Nous).

C'est l'image identitaire produite par Yann de Lyon sur le site Internet Rapnet dédié au Rap qui intervient dans un échange définitoire du Rap (l'orthographe est respecté) :

"Je crois qu'il y a une chose que personne n'a compris (a part 1 ou 2 pers) : Le Hip Hop, c'est notre culture, on l'aime parce qu'elle nous est propre, à nous dans les técis et qu'elle traduit la plupart de nos but. Vous allez vous prendre la tete pour une question de sapes, de traduction du mot rap,... Quand je traine avec les lascar de mon quartier, les seuls questions qui se posent sur le HH sont bases sur les nouveaute underground ou autres. Moi je $m$ 'habille en large, d'autres s'habille en survetement, d'autres en lewis et Ralph Loraine (desole pour l'orthographe), etc.

"En vous disant cela, j'essaye de vous faire comprendre qu'il y a des choses plus importantes que les vetements ou la traduction de certains mots. Tout le monde a son style, le HH accueil tout les styles.

» Notre force téci est dans nos pensees, pas dans nos apparences! (22 novembre 1998).

Le Hip-Hop et le Rap sont ici définis comme culture commune de la cité ( $«$ à nous dans les técis» (verlan de " cité »); " nos buts»; les "lascars de mon quartier»; la "force téci»), et ce dans une conception ouverte qui ne se fonde pas sur une unité de style ou de comportement (une mode vestimentaire, par exemple) mais sur une communauté de pensée minoritaire, les «nouveautés underground» (pour Yann, qui exclut le racisme, le Front National, la haine de la police...). Nous sommes loin d'une «langue qui ne connaît que la proximité et qui fuit avec effroi le large", pour reprendre la catégorisation d'Alain Bentolila. N'est ce pas une citoyenneté qui se construit dans l'argumentation autour des marqueurs identitaires du Hip-Hop à laquelle participe Yann ? (Cf., Annexe 2, p. 00).

\section{L'identité concurrentielle}

L'identité concurrentielle des membres secondaires des cités procède d'une catégorisation autoréférentielle et d'une identification différentielle (Moi $\neq$ Nous).

L'exemple ici, est extrait d'une publication, résultat d'un atelier d'écriture Rap organisé à Marseille pour les enfants du quartier du Panier (écrirons-nous « Kartier » marquant par là le «K» marseillais ?) par le Centre International de Poésie Marseille dans le cadre d'une action nationale du Ministère de la Culture intitulée Saison en Banlieue (1992).

«Le RAP on s'en sert pour sortir notre rage, pour insulter, les gens de la haute société. Qui nous emmerdent. Nous, les BWZ (Black 'nd White Zulus), par rapport aux autres, nous avons des refrains... pas comme les autres. Ils sont un peu entraînants. Sans nous vanter. Nos musiques sont pas les mêmes", (p. 6).

"Nous, on fait pas comme les autres, on va en ville, on tchatche marseillais, on dit ce qu'on

fait et tout. Nous on est plutôt regroupés sur nous-mêmes. On travaille dans notre quartier. 
On est souvent dans notre quartier... On essaie de monter notre rap, on va pas dans tous les concerts voir ce que fait l'autre, les Cabrel, ce que fait l'autre ", (p. 9). catégorisation d'Alain Bentolila qui parle du français des cités comme d'une langue qui "[...] ferme définitivement les portes du monde de l'écrit.»

"Le Mars-Eyes, peace à tous!

Pour tout l'or des mots = Claude Gagnere. Ce livre c'est pour le contenu sur la langue

française et les delires linguistiques que l'on peut faire avec le FRENCH du genre:

La protubérance

du pote Hubert Hanz

La peau tubée, rance

Dupe rot, tube et rance... etc

et c'est pas seulement frenchie-rigolo; en tous les cas les modèles dans ce bouquin inspirent et permettent de structurer la facon d'écrire du rap par exemple en ayant des exercices de rimes, de dictions, de sons et de bizness de la rime. Je pense que c'est utile pour ceux et celles qui veulent poser du rap sur de la musique Hip Hop. " (17 novembre 1998).

En passant à l'écrit, les pratiques langagières des cités n'échappent pas à la structuration, dans une démarche normalisatrice qui ne dédaigne pas les ressources de la rhétorique. Comme le remarque dans l'atelier Nathalie Binisti, même si les jeunes font mine de rejeter le français standard lorsqu'ils sont en groupe et se mettent en scène face aux autres comme des jeunes des quartiers, ils n'en ont pas moins conscience de l'importance de maitriser la forme dominante (notamment en passant au Lycée ou en recherche d'emploi), en représentation au moins ("ce que je dis, je l'écrirais pas pour le prof », P.R., 14 septembre 1997). Ainsi, « avoir la tchatche » signifie à la fois maitriser le parler quartier et le français standard. C'est le fait d'un jeune qui peut se mesurer aux autres dans les joutes verbales mais qui peut aussi éviter une bagarre, par exemple, tout en « préservant sa face» ou embrouiller («rendre fou ») les représentants d'une autorité (professeurs, surveillants, policiers...).

\section{L'identité singularisante}

L'identité singularisante des membres périphériques est déterminée par le processus de catégorisation hétéroréférentielle et d'une identification par similitude (Moi = Eux).

C'est l'image identitaire produite dans un échange internet par un policier amateur de Hip-Hop qui met entre parenthèses son statut («tout n'est pas dans l'uniforme») au profit d'une affirmation de rôle où la citoyenneté n'est plus le fait d'un groupe social particulier dont seraient exclus les jeunes des cités mais une valeur que doivent partager les amateurs d'un style qui privilégie l'être sur l'apparence :

\footnotetext{
- Dee «Je crois être bien placé pour te comprendre car lorsque la liste a su que j'étais policier, ca en a surpris plus d'un. Quoi, Un Cop MC? ?? Eh oui, tout n'est pas dans l'uniforme, car le Hip Hop, ca se vit en dedans, et c'est pas parce que plusieurs rappers et amateurs de rap bullshit la Police que je devrais m'y arrêter car ils
} 
ont raison de dénoncer la brutalité et toute autre forme d'abus, car cela n'a pas sa place dans lapolice. Alors, peace à tout le monde et à Sylvia pour son Keep it real with Hip Hop, car c'est ce qu'il manque à cette culture, des gens qui savent apprécier le Hip Hop sans pour autant se faire une banque pour se payer du Hilfger et une casquette Kangol et parler tout plein de Trash Talk... » (Liste Rapnei, le 3 décembre 1998).

Les nouveaux manuels d'apprentissage reflètent les nouveaux usages de la langue.

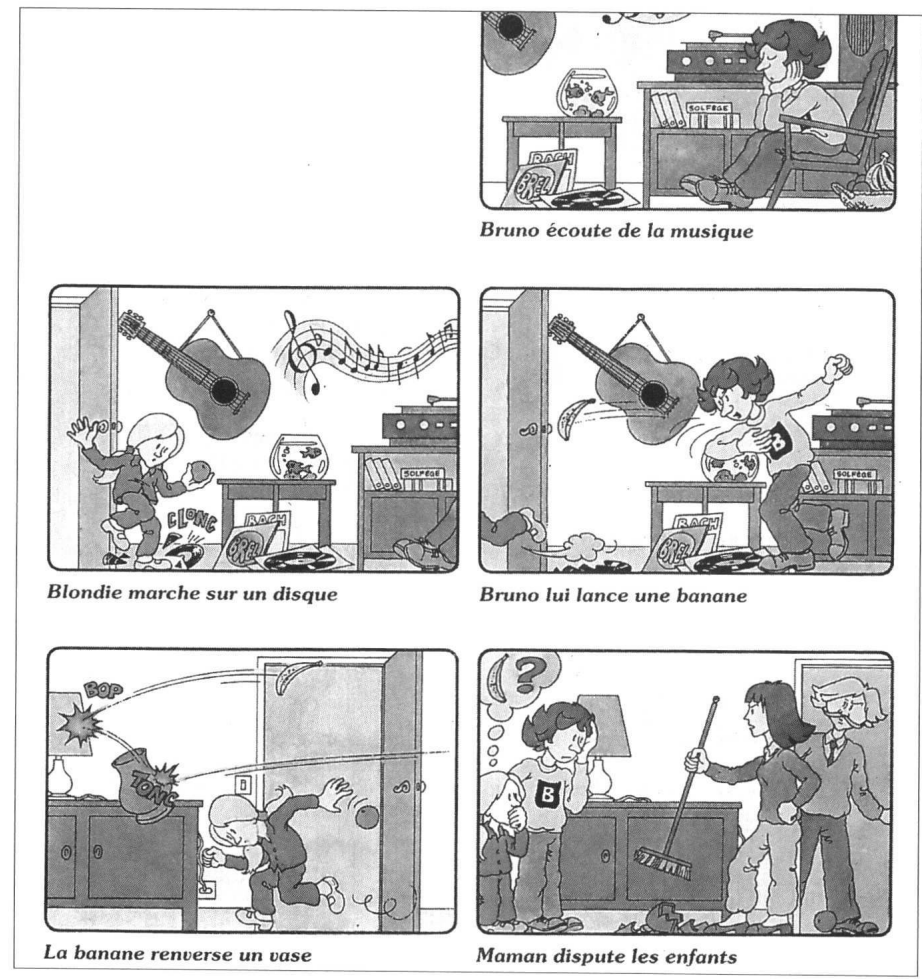

LASCAR S., LECLERCQ P. \& LECLERCQ A. : Mon Copain Bruno - Fin CP et début CE1 (Collection : Vivre par l'écrit). Paris, Magnard, 1982, 63 p., 39.

\section{L'identité insularisante}

L'identité insularisante des membres marginaux résulte d'une catégorisation hétéroréférentielle et d'une identification différentielle (Moi $\neq$ Eux).

Ainsi de l'image identitaire produite par Carlo, un marseillais de la liste Rapnet qui s'exclut du groupe d'appartenance (la "téci») sur des critères moraux (refus de la délinquance), au prix fort (pas d'argent facile) mais en gagnant une citoyenneté économique («citoyen maçon »).

- Carlo "Moi aussi, je suis de la téci. J'en suis sorti en travaillant sérieux (legal) pour une grosse caisse, pas comme les Kramés [ « scarla », « lascar » dans le sens de « délinquant » à Marseille] pouilleux qui font leur 18000 par mois dans le trafic de blanche et se prennent pour des victimes. Moi je fais même pas 7000 mais je comme citoyen maçon mais je me respecte et dégun ["personne" en marseillais] me casse les burnes. (2 décembre 1999). 


\section{En guise de conclusion}

Des débats de l'atelier ressort une double nécessité, à la fois descriptive des pratiques langagières urbaines des jeunes dans leur diversité (parler cité mais aussi usages du français de référence) et des formes de citoyenneté induites par les langues des cités, au delà d'une stigmatisation d'usages "pauvres " («marginalisants", «inaptes à une pratique citoyenne » supposant une " capacité à décontextualiser ») en fonction d'a priori idéologiques: il existerait un «langage des jeunes des cités", conçu comme calque du modèle du « langage des classes populaires », la différenciation n'étant plus uniquement sociale (disparition du prolétariat) mais générationnelle et géographique. Dans ce cadre d'étude, l'école constitue un lieu essentiel d'observation de ce qui se dit et de ce qui se construit ensemble, citoyenneté et langage comme produits de l'action éducative.

Manifestation anti-raciste dans une rue de la ville de Marseille.

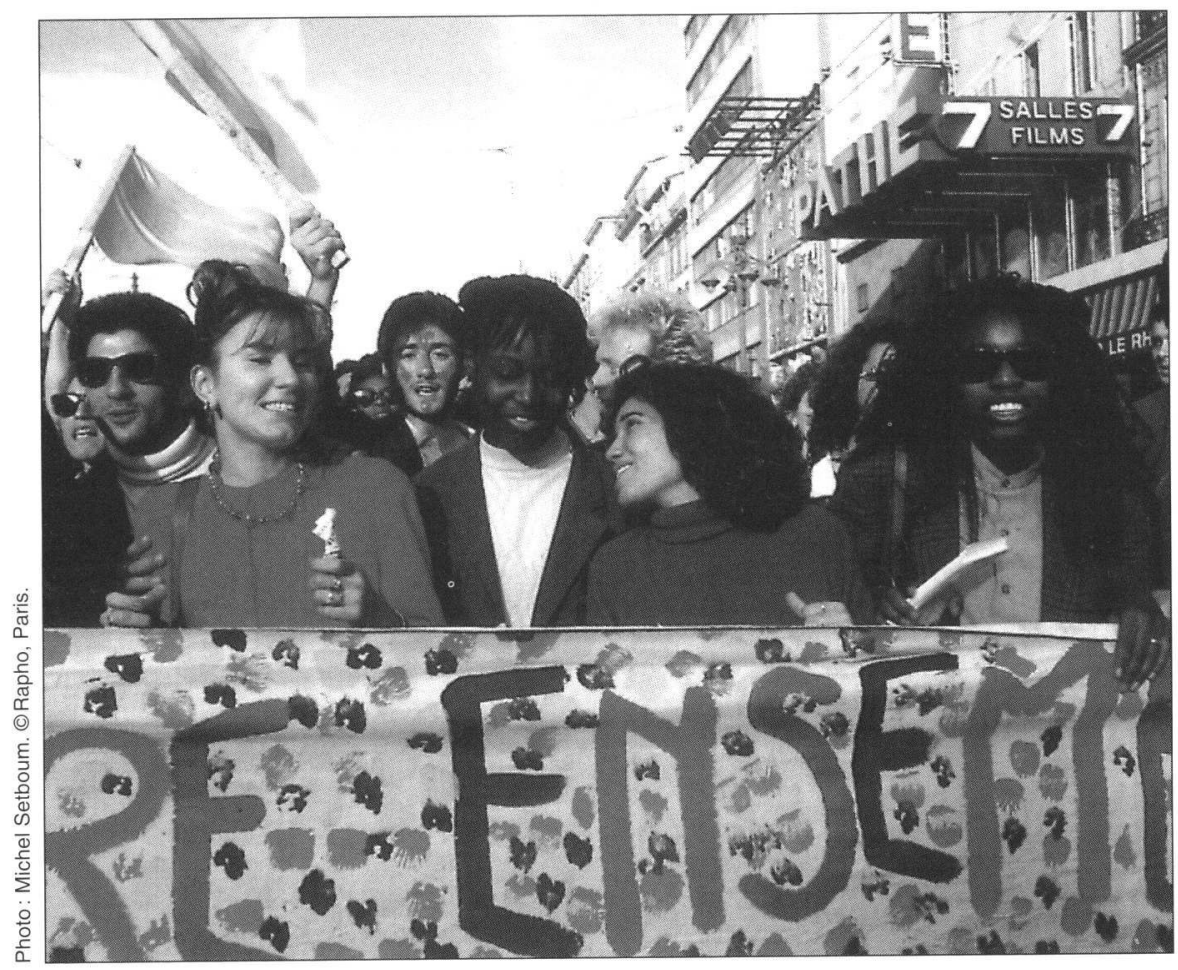

Le Courrier de I'UNESCO. Paris, UNESCO, mars 1997 (Vol. 50, №3), 50 p., p. 27 


\section{BIBLIOGRAPHIE}

BAGGIONI D. : « Les jeunes des cités et restriction des échanges langagiers », in L'école de la deuxième chance, Actes du colloque organisé à Marseille les 9 et 10 décembre 1996. Institut de la Méditerranée, Editions de l'aube, 1997.

BENTOLIA A. : «Les faux-semblants du français branché », in Le Monde, № du 26 mars 1998. Paris, 1998.

BILLIEZ J. : « Le < parler véhiculaire interethnique > de groupes d'adolescents en milieu urbain », in Des villes et des langues, Actes du colloque international de Dakar (15-17 décembre 1990). Paris, Didier Erudition, Collection : Langues et développement, 1990.

BILLIEZ J. et DABENE L. : « Le parler des jeunes issus de l'immigration », in Vermes G. et Boutet J. (dir) : France, pays multilingue - tome 2 : « pratiques des langues de France ». Paris, L'Harmattan, Collection : Logiques sociales, 1987.

BINISTT N. : Le parler des jeunes dans les quartiers Nord de Marseille, mémoire de maîtrise de Sciences du Langage sous la direction de Jean Doneux. Université de Provence, 1997.

BINISTI N. : Les filles d'la Soli et leur franc parler... - Une étude sur les pratiques langagières de jeunes filles habitant dans une cité des quartiers nord de Marseille, mémoire de DEA de Sciences du Langage, sous la direction d'Alain Giacomi. Université de Provence, 1998.

BOURDIEU P. : Ce que parler veut dire. Paris, Fayard, 1982.

BOURDIEU P. : « Vous avez dit 〈populaire〉? », in Actes de la Recherche en Sciences Sociales, № 46 (mars 1983). 1983.

BOYER H. : « Le jeune tel qu'on en parle », in Langage et société, № 70. 1994.

BOYER H. : "Conflits d'usages, conflits d'images », in BOYER H. (éd.) : Plurilinguisme : " contact » ou « conflit » de langues ?. Paris, L'Harmattan, Collection : Sociolinguistique, 1997.

BRANCA-ROSOFF S. : «Les imaginaires de langues », in BOYERH. (dir.) : Sociolinguistique :

territoire et objets. Delachaux et Niestlé, 1996.

CALVET L.-J. : Les voix de la ville - Introduction à la sociolinguistique urbaine. Paris, Payot, Collection : Essais, 1994.

CASTELLANI G. : « Langage des banlieues, malentendu et violence scolaire », in SKOLE - Cahiers de la recherche et du développement, Actes du colloque « Touche pas à ma langue ! [...] - Les langages des banlieues », (IUFM de Marseille, 26-28 septembre 1996). Marseille, IUFM de Marseille, 1997.

DEPÉTRIS J.-P. (éd) : Une Saison au Panier II, Spécial Rap. Marseille, Imprimerie du Collège, 1992. GARCIA D. et MALAURIE G. : «Verlan cherche deuxième souffle » (entretiens avec Alain Rey, Henriette Walter et Pierre Merle), in Le Nouvel Observateur. Paris, 1998.

GOUDAILLIER J.-P. : Comment tu tchatches ! Dictionnaire du français contemporain des cités. Maisonneuve et Larose, 1997.

IZZO J.-C. : « Oui, mais quel mot, Marseille », in ARMOGATHEet KASBARIAN : Dico Marseillais. Jeanne Laffite, 1998. 
KASBARIAN J.-M. : « Quelques repères pour décrire les ‘ langages des banlieues > ", in SKOLE Cahiers de la recherche et du développement, Actes du colloque « Touche pas à ma langue ! [...] Les langages des banlieues ", (IUFM de Marseille, 26-28 septembre 1996). Marseille, IUFM de Marseille, 1997. LABOV W. : Le parler ordinaire. Paris, Minuit, 1978.

LAKS B. : « Langage et pratiques sociales - Etude sociolinguistique d'un groupe d'adolescents », in Actes de la Recherche en Sciences Sociales, № 46 (mars 1983). 1983. LEPOUTRE D. : Cour de banlieue - Codes, rites et langages. Paris, Odile Jacob, 1997. LOPEZ J. A. : Langage et identité cultuelle dans un quartier de Montpellier : La Paillade, mémoire de maîtrise de F.L.E., sous la direction de Jean-Marie Prieur. Montpellier, Université Paul Valéry (Montpellier III), 1996.

LOPEZ J.A. : Grillades, enfle et baratin - Formes ritualisées de communication chez les jeunes Pailladins, mémoire de DEA en Sciences du Langage, dirigé par Jacques Brès et Jean-Marie Prieur. Montpellier, Université Paul Valéry (Montpellier III), 1998.

\section{ANNEXES}

\section{Annexes}

\section{- Annexe 1 -}

Titres des livres et articles analysés - ( $C f$. , diagramme et tableau, p. 89)

AGUILLOU P. et SAIKI N. : La téci à Panam. Paris, Michel Lafon, 1996.

[DL : (3) - CG :(ø) - T : (6)]

BEZARD C. : « Le langage des jeunes : à décrypter avec décodeur », in L'événement du jeudi, № 457. Paris, 1993.

[DL :(2) - CG :(5) - T : (ø)]

DEMOUGEOT M., DUVILLARD J., LAURIOZ H. et MARCOZ L. : Nouveau français : la compii Petit dico des mots interdits aux parents. Paris, J.C. Lattès, 1994.

[DL : (4) - CG : (5) - T : (ø)]

EPSTEIN J. : Petit guide de conversation usuelle pour changer le Monde sans fatigue.

Editions Universitaires, 1987.

$[\mathrm{DL}:(4)-\mathrm{CG}:(\varnothing)-\mathrm{T}:(\varnothing)]$

GARCIA D. : « Faut-il vraiment un interprète ? ", in Le Nouvel Observateur - (banlieue

tchatche), № 1771 (15/10/1998). Paris, 1998.

[DL : (3) - CG : (ø) - T :(7)]

GIRARD E. et KERNEL B. : Ado/parents, le manuel - Guide de conversation. Paris, Presses de la Cité, 1994.

[DL : (2) - CG : (5) - T :(ø)]

GOUDAILLIER J-P : Comment tu tchatches ! Dictionnaire du français contemporain des cités.Maisonneuve et Larose, 1997. 
[DL : (4) - CG : (ø) - T : (6)]

GRASSIN S. : « Réservé aux moins de 20 ans », in L'Express, № 1728. Paris, 1984.

[DL :(ø) - CG : (5) - T :(ø)]

HERNANDEZ F. : Panique ta langue. Paris, Editions du Rocher, 1996.

[DL : (1) - CG : (ø) - T :(ø)]

MAZURE D. : « Savez-vous parler le jeune », in L'Echo des Savanes, № 128 (juin). Paris, 1994.

[DL : (ø) - CG : (5) - T :(ø)]

MERLE E : Dictionnaire du français branché.Paris, Seuil, 1986.

[DL : (4) - CG : (5) - T :(ø)]

MERLE E : Le dico du français qui se cause.Éditions Milan, 1998.

[DL : (4) - CG :(ø) - T :(ø)]

OBALS H., SORAL A. et PASCHE A. : Les mouvements de mode expliqués aux parents. Paris, Robert Laffont, 1984.

$[\mathrm{DL}:(\varnothing)-\mathrm{CG}:(5)-\mathrm{T}:(\varnothing)]$

PIERRE-ADOLPHE P., MAMOUD M. et TZANOS : Le dico de la banlieue. Paris, La Sirène, 1995.

[DL : (1) - CG : (ø) - T :(7)]

RODRIGUE V. : « Le jargon interdit aux parents », in 20 ans, № 15. Paris, 1987.

[DL : (3) - CG : (5) - T : (ø)]

SCHIFRES A. : « Le jeune tel qu'ils le parlent », in Le Nouvel Observateur, № du 17-23 mars. Paris, 1982.

[DL : (ø) - CG : (5) - T : (ø)]

SÉGUIN B. et TEILLARD F. : Les céfrans parlent aux français. Paris, Calman-Lévy, 1996.

[DL : (ø) - CG : (ø) - T : (ø)]

VANDEL P. : Le dico français/français. Paris, J.C. Lattès, 1993.

[DL : (4) - CG : (ø) - T : (ø)]

VANDEL E : «Le jeune tel qu'ils le parlent », in Le Nouvel Observateur, № du 17-23 mars. Paris, 1994.

[DL :(ø)-CG :(5)-T :(ø)]

VERDELHAN-BOURGADE M. : « Parlez-vous branché ?», in Europe, № 738. 1990.

[DL : (4) - CG : (ø) - T : (ø)] 


\section{- Annexe 2 -}

\section{Échange internet (liste Rapnet) du 22 au 27 novembre 1998}

(l'orthographe est celle des intervenants dans le forum)

\section{Pour tous :}

"De Sylvia, salut à tous. Corment s'habille un vrai rapper? C'est clair : Nike, Fila, Pelle-Pelle, Tommy Hil, South Pole, Phat Farm, rien que des marques quoi, et surtout : baggy-style, c-à-d toujours XXL. Un extrait d'un article du Nouvel Obs, intitulé Génération Hip Hop te l'explique : Baggy(pantalon ultralarge) qui tombe sur les grosses baskets (les classiques Nike - Reebok - Fila ou les plus féminines No Name), énormes doudounes ou grands coupe-vent empruntés aux skippers suédois (Helly Hansen ou South Pole). Ici comme ailleurs, ce qui compte c'est la marque. En vogue cette saison : la griffe Tommy Hilfiger, qui décline tout l'uniforme du parfait rappeur, du bonnet ou de la casquette (portée visière sur la nuque) à la parka. Pour les plus pointus, des accessoires clinquants. Grosses chaînes, pendentifs, ceintures à grosses boucles et même... fausses dents en or (200 francs) !> Voilà, les instruction essentielles résumées en qqes lignes... "

\section{Intervenant 1}

«Écoute : je me fous de toi (j'espère que tu ne m'en veux pas...), cet article, c'est des conneries : un rapper s'habille comme il se sent. Les trucs que cet article cite, sont bien des accessoires de rappers qui font partie d'une espèce de code, certes, mais je le répète et le répète et le répèterai toujours: reste authentique! Mets ce que tu veux, tout dépend de ce que tu veux exprimer.

Moi, par ex. il y a des jours où je me promène en survêt, d'autres où je suis baggy, d'autres où je suis fringuée en 'fille respectable et parfoisj'ai le look ethno, peu importe, tant que tu sais et n'oublie pas qui tu es.»

\section{Intervenant 2}

"Hé Sylvia, je suis ABSOLUMENT de l'avis de ton avis: ce que tu dis est bourré de sens et de vérité. Il n'y a pas besoin d'être fringué South Pole ou de parler le verlan pour savoir ce qu'est le Hip Hop. Les fringues et le langage sont des trucs qui rallient, mais qui ne sont pas nécessaires; je juge beaucoup plus important d'être authentique, de (practice what you preach), et de conserver son propre style, quoiqu'il arrive. » ETIENNE

\section{Intervenant 3}

"Nice Man in love. Un vrai rapper peut s'habiller comme il veut car la culture Hip Hop, c'est un état d'esprit et non une parade de mode. ...Tu peux faire du Hip Hop même avec un uniforme bleu et un Magnum sur la cuisse... »

\section{Intervenant 4}

«À propos de rap, de Hip Hop, et de technique : à votre avis, ou est situé MC Solaar dans tout ca? J'aime ce qu'il fait, mais quand je compare à IAM, par ex, c'est pas vraiment le même chose... J'ai lu un article qui lui reprochait de s'éloigner du HH des cités et de se rapprocher de la chanson française genre Gainsbourg. Reproche que je trouve complètement wack parce qu'il n'a aucune obligation vis-à-vis de la cité... Mais toujours est-il qu'il est spécial. Qu'en pensez-vous? Comment est-ce qu'on le considère en France? "J.B.

\section{Intervenant 5}




\begin{abstract}
«Join me! Salut man!!
"En ce qui me concerne, je considere MC Solaar comme un des premiers (il y en a quelques autres) qui aient lancer ce style de musique en france. Ce pelo est extremement intelligent et de plus, il s'occupe de gamins defavorises dans les quartiers. C'est vrai qu'il s'ait fait tailler par d'autres rappeur pour son style de musique qui etait soit disant trop vieux, pas dans la mode,... Mais c'est quand meme lui qui ma fait decouvrir un style de musique, j'aime et je respecte sa musique. »
\end{abstract} RICK the red!

\title{
Intervenant 6
}

"Je crois qu'il y a une chose que personne n'a compris (a part 1 ou 2 pers): Le Hip Hop, c'est notre culture, on l'aime parce qu'elle nous est propre, à nous dans les técis et qu'elle traduit la plupart de nos but. Vous allez vous prendre la tete pour une question de sapes, de traduction du mot rap, Quand je traine avec les lascar de mon quartier, les seuls questions qui se posent sur le $\mathrm{HH}$ sont bases sur les nouveaute underground ou autres. Moi je m'habille en large, d'autres s'habille en survetement, d'autres en lewis et Ralph Loraine (desole pour l'orthographe), etc.

"En vous disant cela, j'essaye de vous faire comprendre qu'il y a des choses plus importantes que les vetements ou la traduction de certains mots. Tout le monde a son style, le HH accueil tout les styles.

« Notre force téci est dans nos pensees, pas dans nos apparences!» YANN de LYON

\section{Intervenant 7}

"Quelle semaine sur la liste! Je suis contente que le unsubscribe ne marche pas!! Je n'ai pas tout bien suivi,sur le racisme et sur le débat HH rap,maisj'aime bien cet esprit de discussion,ça fait aussi partie du mouvement !! Pour donner une derniere metaphore, le Hh c'est un corps tout entier, et le rap par ex, c'est la tête, la bouche, les oreilles... le DJing et le graf c'est la tête et les mains... la break, c'est les membres et plein d'autres qualités!!» SYLVIA

\section{NOTES}

1. On souscrira au constat de Billiez et Dabène $(1987$, p. 62$)$ pour qui « si les recherches ne manquent pas dans ce domaine, elles sont surtout le fait des spécialistes des sciences sociales ou économiques. Les linguistes ne semblent pas s'y être sérieusement intéressés - si ce n'est pour étudier l'acquisition, par les migrants, de la langue du pays d'accueil».

2. Objet «sérieux» de la nouvelle science dont Saussure revendique la paternité, la langue comme système doit être décrite en synchronie indépendamment de l'activité des sujets, de la parole des locuteurs qui l'actualisent dans différents contextes soumis à des contraintes extralinguistiques qu'il s'agit de mettre entre parenthèses dans une approche objective. La dichotomie primitive langue/parole a, depuis, servi de cadre commode de catégorisation historique, sociale, psychologique entre états de langue auxquels se trouvent attachés des jugements de valeur. On retrouvera ainsi les oppositions entre oral et écrit, spontané et stylisé, libre et prescriptif, relâché et soutenu, dialectal et national, expressif et cognitif. La dichotomie langue/langage se trouve de fait bornée sociologiquement par l'opposition entre populaire et bourgeois d'une part, naturel et cultivé d'autre part. Plus récemment, soumise à la question de l'actualité (question urbaine de la violence des jeunes qui serait aussi «violence faite au français »), la classique dichotomie resurgit dans le débat entre «langues des cités » et «langue de scolarisation », légitimité de la communication ordinaire comme sociolecte différentiateur, 
constituant des identités territorialisées et normativité du français scolaire, unifiant, constitutif d'une communauté linguistique et d'une identité nationale abstraites.

3. Liée selon Lepoutre au mode de diffusion médiatique (1997, p. 102) : «Les journalistes ne sont d'ailleurs pas spécialement en cause, mais c'est plutôt la logique spécifique de leur champ professionnel qui détermine dans une large mesure la forme et le contenu des reportages".

4. Évoquée par P Merle (in - GARCIA et MALAURIE : 1988, p. 32) en ces termes : «[...] que les jeunes cherchent à se démarquer par le langage n'est pas nouveau. La grande différence, c'est que leurs inventions sont immédiatement aspirées par les médias et la pub».

5. Si l'existence de variétés urbaines n'est bien évidemment pas nouvelle, on soulignera avec Castellani (1997, p. 120) que « [...] la nouveauté, c'est qu'il [le parler des banlieues, des quartiers] est de plus en plus perçu comme une agression tant par ceux qui ne le comprennent pas que par ceux qui ont peur de le comprendre ».

6. Nathalie Binisiti remarque dans la discussion que la plupart des études concernant la « langue des Cités » soulignent la forte valorisation de la parole chez les jeunes. En effet, quelqu'un qui «a la tchatche » sera quelqu'un d'admiré et de respecté.

\section{RÉSUMÉS}

L'école est confrontée aux pratiques langagières urbaines quand la langue de scolarisation, langue unifiante, doit faire face, avec, le français des cités des élèves. Quelles approches sont à développer en classe pour éviter deux attitudes inefficaces, stigmatisation ou survalorisation de la réalité langagière des élèves des cités?

Schools are confronted with the challenge of urban (ghetto) language usage whenever the language of schooling, society's common language, must deal head on with the student's urban language forms. Which approaches should be developed in class towards the concrete reality of the language spoken by urban (ghetto) students, in order to avoid the two ineffective attitudes of stigmatizing this language or of pandering to it?

\section{INDEX}

Mots-clés : identité, norme langagière, variation sociale

Keywords : identity, Language norm, social variation

\section{AUTEUR}

JEAN-MICHEL KASBARIAN

Université de Provence 